Supplement of Biogeosciences, 17, 6457-6474, 2020

https://doi.org/10.5194/bg-17-6457-2020-supplement

(C) Author(s) 2020. This work is distributed under

the Creative Commons Attribution 4.0 License.

(c) (1)

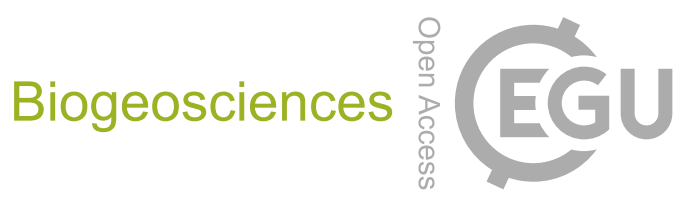

Supplement of

\title{
Vertical mobility of pyrogenic organic matter in soils: a column experi-
} ment

Marcus Schiedung et al.

Correspondence to: Samuel Abiven (abiven@ biotite.ens.fr)

The copyright of individual parts of the supplement might differ from the CC BY 4.0 License. 

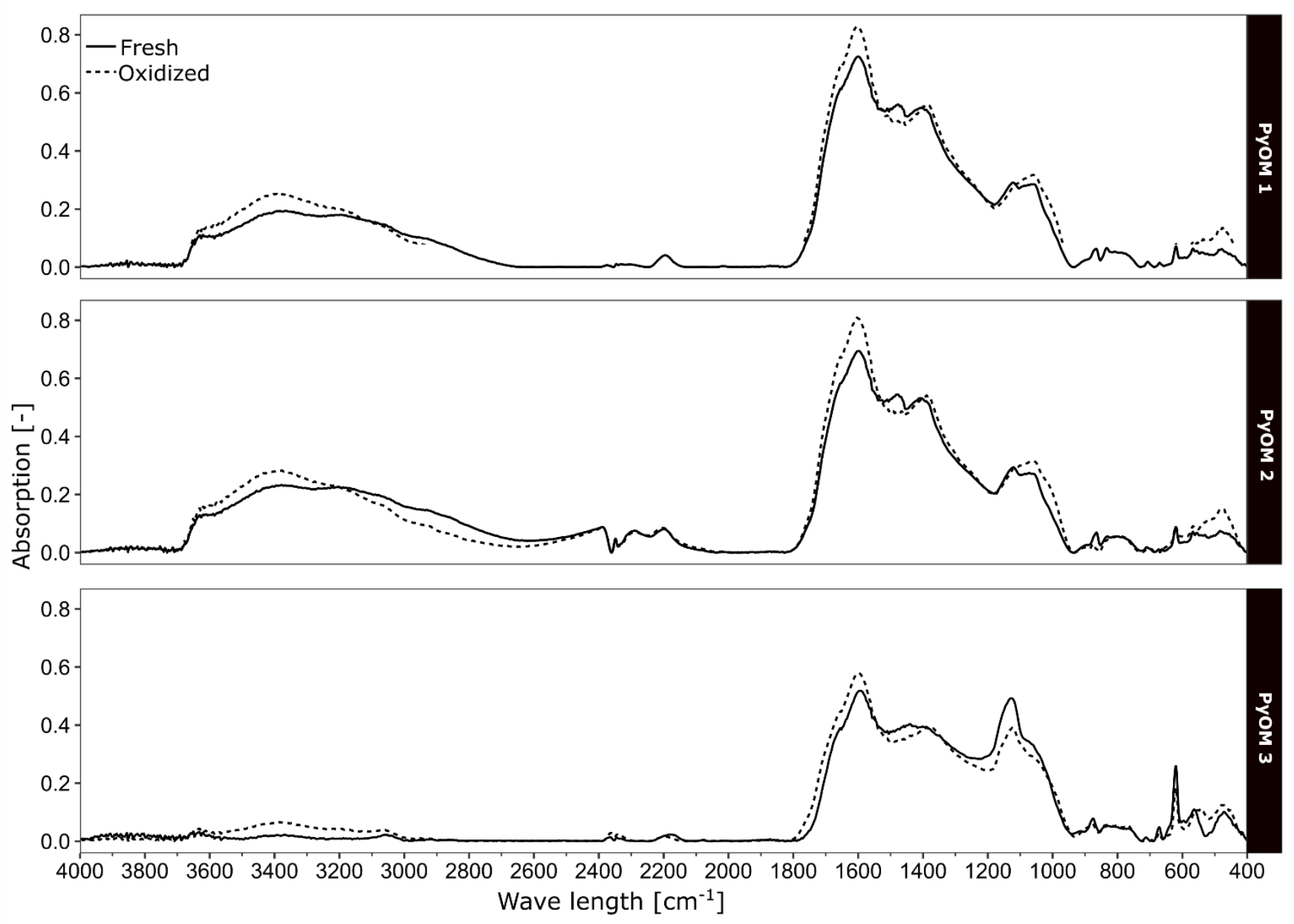

Figure S1: Full DRIFT spectra of all three used fresh and oxidized PyOM. For details see figure 1 and table 2. 


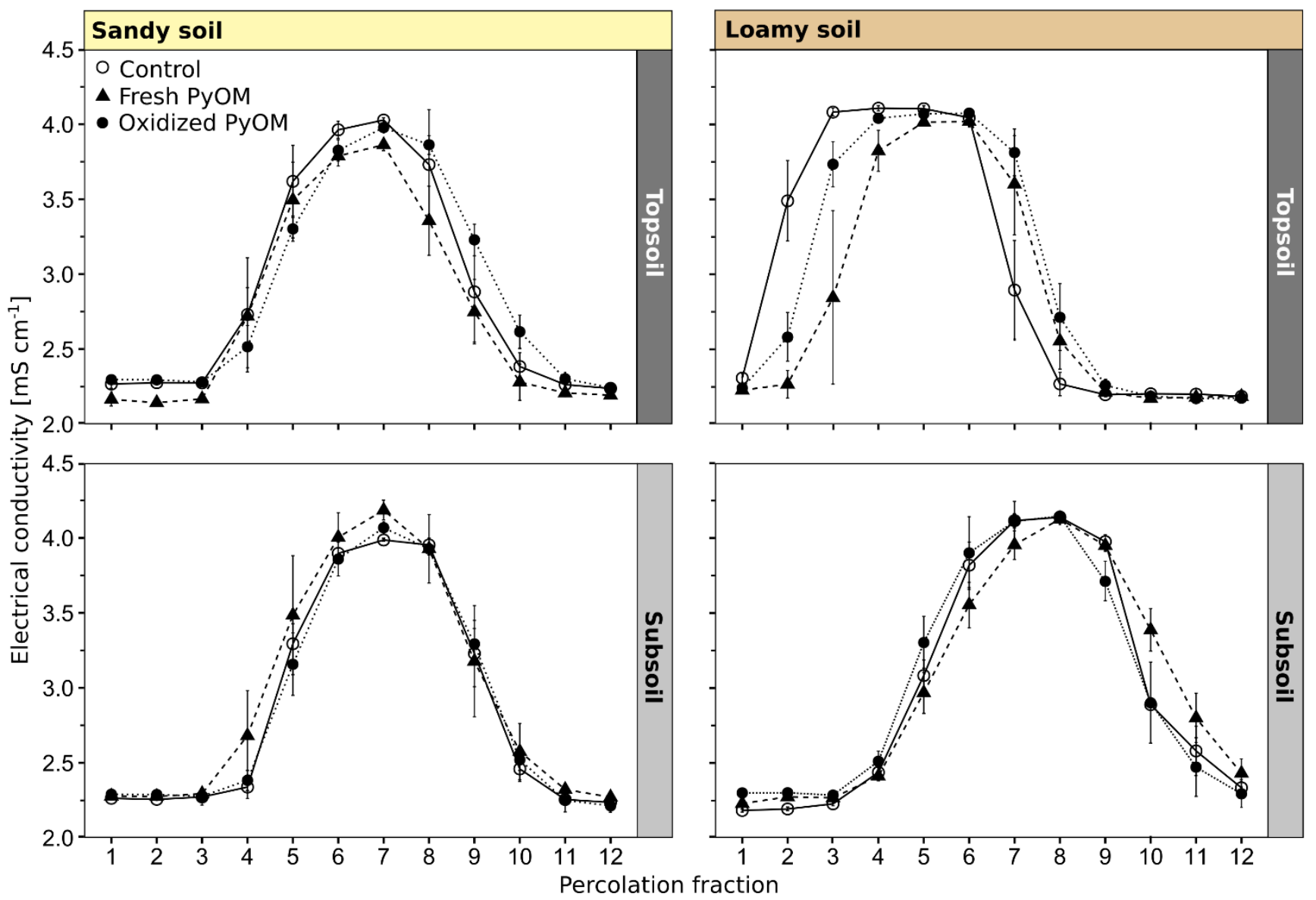

5 Figure S2: Breakthrough curves (BTC) for topsoil and subsoil of the sandy and loamy soil as average of control columns and columns with fresh and oxidized PyOM addition $( \pm 1 \mathrm{SE})$. The percolation fraction represent each a percolation for 6 minutes with a flow of

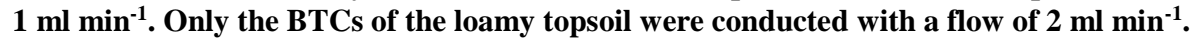


Table S1: Diffusion coefficients $D$, convective velocity $v$ and dispersivities $\lambda$ fitted for all columns for sandy and loamy soil.

\begin{tabular}{|c|c|c|c|c|}
\hline & $\begin{array}{l}\text { Column } \\
\text { replicate }\end{array}$ & $\begin{array}{c}v \\
{\left[\mathrm{~cm} \mathrm{~min}^{-1}\right]}\end{array}$ & $\begin{array}{c}\boldsymbol{D} \\
{\left[\mathrm{cm}^{2} \mathrm{~min}^{-1}\right]}\end{array}$ & $\begin{array}{c}\lambda \\
{[\mathrm{cm}]}\end{array}$ \\
\hline \multicolumn{5}{|l|}{ Sandy topsoil } \\
\hline \multirow[t]{4}{*}{ Control } & 1 & 0.31 & 0.036 & 0.12 \\
\hline & 2 & 0.36 & 0.035 & 0.10 \\
\hline & 3 & 0.31 & 0.027 & 0.09 \\
\hline & 4 & 0.30 & 0.030 & 0.09 \\
\hline \multirow[t]{4}{*}{ Fresh PyOM } & 1 & 0.31 & 0.038 & 0.12 \\
\hline & 2 & 0.33 & 0.053 & 0.16 \\
\hline & 3 & 0.34 & 0.041 & 0.12 \\
\hline & 4 & 0.29 & 0.039 & 0.13 \\
\hline \multirow[t]{4}{*}{ Oxidized PyOM } & 1 & 0.28 & 0.057 & 0.21 \\
\hline & 2 & 0.29 & 0.033 & 0.12 \\
\hline & 3 & 0.29 & 0.031 & 0.11 \\
\hline & 4 & 0.31 & 0.057 & 0.19 \\
\hline \multicolumn{5}{|l|}{ Sandy subsoil } \\
\hline \multirow[t]{4}{*}{ Control } & 1 & 0.28 & 0.030 & 0.10 \\
\hline & 2 & 0.29 & 0.024 & 0.08 \\
\hline & 3 & 0.28 & 0.025 & 0.09 \\
\hline & 4 & 0.31 & 0.020 & 0.08 \\
\hline \multirow[t]{4}{*}{ Fresh PyOM } & 1 & 0.27 & 0.041 & 0.15 \\
\hline & 2 & 0.27 & 0.058 & 0.21 \\
\hline & 3 & 0.33 & 0.076 & 0.23 \\
\hline & 4 & 0.31 & 0.056 & 0.18 \\
\hline \multirow[t]{4}{*}{ Oxidized PyOM } & 1 & 0.27 & 0.023 & 0.09 \\
\hline & 2 & 0.28 & 0.037 & 0.13 \\
\hline & 3 & 0.29 & 0.029 & 0.10 \\
\hline & 4 & 0.28 & 0.050 & 0.18 \\
\hline
\end{tabular}

10 
Table S1: continued

\begin{tabular}{|c|c|c|c|c|}
\hline & $\begin{array}{l}\text { Column } \\
\text { replicate }\end{array}$ & $\begin{array}{c}v \\
{\left[\mathrm{~cm} \mathrm{~min}^{-1}\right]}\end{array}$ & $\begin{array}{c}\boldsymbol{D} \\
{\left[\mathrm{cm}^{2} \mathrm{~min}^{-1}\right]}\end{array}$ & $\begin{array}{c}\lambda \\
{[\mathrm{cm}]} \\
\end{array}$ \\
\hline \multicolumn{5}{|l|}{ Loamy topsoil } \\
\hline \multirow[t]{4}{*}{ Control } & 1 & 0.72 & 0.326 & 0.46 \\
\hline & 2 & 0.77 & 0.081 & 0.10 \\
\hline & 3 & 0.85 & 0.318 & 0.37 \\
\hline & 4 & 0.71 & 0.310 & 0.44 \\
\hline \multirow[t]{4}{*}{ Fresh PyOM } & 1 & 0.50 & 0.271 & 0.54 \\
\hline & 2 & 0.54 & 0.097 & 0.18 \\
\hline & 3 & 0.50 & 0.116 & 0.23 \\
\hline & 4 & 0.42 & 0.053 & 0.13 \\
\hline \multirow[t]{4}{*}{ Oxidized PyOM } & 1 & 0.49 & 0.121 & 0.25 \\
\hline & 2 & 0.63 & 0.142 & 0.23 \\
\hline & 3 & 0.54 & 0.141 & 0.26 \\
\hline & 4 & 0.53 & 0.142 & 0.27 \\
\hline \multicolumn{5}{|l|}{ Loamy subsoil } \\
\hline \multirow[t]{4}{*}{ Control } & 1 & 0.28 & 0.027 & 0.10 \\
\hline & 2 & 0.27 & 0.042 & 0.15 \\
\hline & 3 & 0.26 & 0.039 & 0.15 \\
\hline & 4 & 0.28 & 0.030 & 0.12 \\
\hline \multirow[t]{4}{*}{ Fresh PyOM } & 1 & 0.27 & 0.041 & 0.15 \\
\hline & 2 & 0.25 & 0.065 & 0.26 \\
\hline & 3 & 0.24 & 0.056 & 0.24 \\
\hline & 4 & 0.25 & 0.050 & 0.20 \\
\hline \multirow[t]{4}{*}{ Oxidized PyOM } & 1 & 0.39 & 0.229 & 0.59 \\
\hline & 2 & 0.29 & 0.036 & 0.12 \\
\hline & 3 & 0.29 & 0.029 & 0.10 \\
\hline & 4 & 0.29 & 0.040 & 0.14 \\
\hline
\end{tabular}


Table S2: pH, EC, percolated native soil organic carbon (nSOC), excess isotope-amount fraction $x E\left({ }^{13} C\right)$, PyOM-C and proportion

\begin{tabular}{|c|c|c|c|c|c|c|c|}
\hline & $\begin{array}{c}\text { Percolation } \\
\qquad\left[1 \mathrm{~m}^{-2}\right] \\
\end{array}$ & $\begin{array}{c}\mathbf{p H} \\
{[-]} \\
\end{array}$ & $\begin{array}{c}\text { EC } \\
{\left[\mathrm{mS} \mathrm{cm}^{-1}\right]}\end{array}$ & $\begin{array}{c}\text { Percolated } \\
\text { nSOC } \\
{\left[\mathrm{mg} \mathrm{l}^{-1}\right]} \\
\end{array}$ & $\begin{array}{c}x E\left({ }^{13} C\right) \\
{[\%]}\end{array}$ & $\begin{array}{c}\text { PyOM-C } \\
{\left[\mu \mathrm{g} \mathrm{l}^{-1}\right]} \\
\end{array}$ & $\begin{array}{c}\text { PyOM-C: } \\
\text { perc. TOC } \\
{[\%]} \\
\end{array}$ \\
\hline \multicolumn{8}{|l|}{ Sandy topsoil } \\
\hline \multirow{5}{*}{ Control } & 1,000 & $3.42(0.02)$ & $2.30(0.01)$ & $12.12(0.24)$ & & & \\
\hline & 3,000 & $3.70(0.04)$ & $2.25(0.01)$ & $4.12(0.07)$ & & & \\
\hline & 8,000 & $4.05(0.01)$ & $2.21(0.01)$ & $1.51(0.03)$ & & & \\
\hline & 13,000 & $4.25(0.02)$ & $2.24(0.01)$ & $1.51(0.13)$ & & & \\
\hline & 18,000 & $4.34(0.02)$ & $2.27(0.01)$ & $0.68(0.09)$ & & & \\
\hline \multirow{5}{*}{ Fresh PyOM } & 1,000 & $3.72(0.03)$ & $2.48(0.01)$ & 45.54 (7.69) & $0.070(0.008)$ & $997.0(72.0)$ & $2.4(0.3)$ \\
\hline & 3,000 & $3.95(0.02)$ & $2.23(0.01)$ & $4.67(0.30)$ & $0.037(0.002)$ & $57.7(8.2)$ & $1.3(0.1)$ \\
\hline & 8,000 & $4.18(0.04)$ & $2.23(0.01)$ & $2.04(0.33)$ & $0.017(0.001)$ & $12.0(3.4)$ & $0.6(0.1)$ \\
\hline & 13,000 & $4.41(0.02)$ & $2.24(0.01)$ & $1.69(0.19)$ & $0.013(0.002)$ & $6.9(0.5)$ & $0.4(0.1)$ \\
\hline & 18,000 & $4.51(0.03)$ & $2.24(0.01)$ & $1.32(0.19)$ & $0.015(0.001)$ & $6.3(0.9)$ & $0.5(0.1)$ \\
\hline \multirow{5}{*}{ Oxidized PyOM } & 1,000 & $3.62(0.02)$ & $2.51(0.01)$ & $28.16(2.09)$ & $0.263(0.010)$ & $2,862.9(319.5)$ & $9.2(0.5)$ \\
\hline & 3,000 & $3.93(0.02)$ & $2.28(0.01)$ & $4.45(0.14)$ & $0.104(0.007)$ & $166.2(8.7)$ & $3.7(0.2)$ \\
\hline & 8,000 & $4.18(0.02)$ & $2.25(0.01)$ & $1.71(0.08)$ & $0.050(0.002)$ & $30.0(1.8)$ & $1.8(0.1)$ \\
\hline & 13,000 & $4.36(0.01)$ & $2.24(0.01)$ & $1.35(0.19)$ & $0.030(0.005)$ & $13.4(1.7)$ & $1.0(0.2)$ \\
\hline & 18,000 & $4.51(0.01)$ & $2.26(0.01)$ & $0.90(0.10)$ & $0.030(0.002)$ & $9.4(1.2)$ & $1.1(0.1)$ \\
\hline \multicolumn{8}{|l|}{ Sandy subsoil } \\
\hline \multirow{5}{*}{ Control } & 1,000 & $4.38(0.02)$ & $2.35(0.02)$ & $3.28(0.18)$ & & & \\
\hline & 3,000 & $4.63(0.04)$ & $2.32(0.01)$ & $0.61(0.04)$ & & & \\
\hline & 8,000 & $4.88(0.04)$ & $2.24(0.01)$ & $0.74(0.06)$ & & & \\
\hline & 13,000 & $5.20(0.05)$ & $2.25(0.01)$ & $0.65(0.21)$ & & & \\
\hline & 18,000 & $5.40(0.03)$ & $2.23(0.01)$ & $1.08(0.13)$ & & & \\
\hline \multirow{5}{*}{ Fresh PyOM } & 1,000 & $4.98(0.07)$ & $2.52(0.02)$ & $13.31(1.55)$ & $0.183(0.008)$ & $1,114.7(169.5)$ & $6.8(0.3)$ \\
\hline & 3,000 & $5.06(0.03)$ & $2.25(0.01)$ & $1.57(0.04)$ & $0.073(0.003)$ & $50.1(3.4)$ & $2.7(0.1)$ \\
\hline & 8,000 & $5.41(0.04)$ & $2.26(0.01)$ & $0.57(0.06)$ & $0.045(0.002)$ & $10.8(0.4)$ & $1.7(0.1)$ \\
\hline & 13,000 & $5.43(0.04)$ & $2.24(0.01)$ & $1.61(0.21)$ & $0.030(0.001)$ & $20.6(2.1)$ & $1.1(0.1)$ \\
\hline & 18,000 & $5.74(0.03)$ & $2.20(0.01)$ & $1.14(0.19)$ & $0.008(0.003)$ & $3.6(1.2)$ & $0.3(0.1)$ \\
\hline \multirow{5}{*}{ Oxidized PyOM } & 1,000 & $4.96(0.07)$ & $2.58(0.02)$ & $11.88(0.51)$ & $0.463(0.018)$ & $2,836.9(166.1)$ & $17.2(1.0)$ \\
\hline & 3,000 & $4.96(0.05)$ & $2.29(0.01)$ & $1.42(0.14)$ & $0.162(0.006)$ & $105.8(8.6)$ & $6.0(0.3)$ \\
\hline & 8,000 & $5.19(0.04)$ & $2.23(0.01)$ & $0.86(0.13)$ & $0.082(0.009)$ & $31.6(6.4)$ & $3.0(0.4)$ \\
\hline & 13,000 & $5.36(0.03)$ & $2.25(0.01)$ & $0.73(0.08)$ & $0.056(0.003)$ & $18.3(2.9)$ & $2.1(0.1)$ \\
\hline & 18,000 & $5.58(0.01)$ & $2.23(0.01)$ & $0.67(0.09)$ & $0.056(0.005)$ & $16.3(1.9)$ & $2.0(0.2)$ \\
\hline
\end{tabular}




\begin{tabular}{|c|c|c|c|c|c|c|c|}
\hline & $\begin{array}{c}\text { Percolation } \\
\qquad\left[1 \mathrm{~m}^{-2}\right] \\
\end{array}$ & $\begin{array}{c}\mathbf{p H} \\
{[-]} \\
\end{array}$ & $\begin{array}{c}\text { EC } \\
{\left[\mathrm{mS} \mathrm{cm}^{-1}\right]}\end{array}$ & $\begin{array}{c}\text { Percolated } \\
\text { nSOC } \\
{\left[\mathrm{mg} \mathrm{l}^{-1}\right]} \\
\end{array}$ & $\begin{array}{c}x E\left({ }^{13} C\right) \\
{[\%]}\end{array}$ & $\begin{array}{c}\text { PyOM-C } \\
{\left[\mu \mathrm{g} \mathrm{l}^{-1}\right]} \\
\end{array}$ & $\begin{array}{c}\text { PyOM-C: } \\
\text { perc. TOC } \\
{[\%]}\end{array}$ \\
\hline \multicolumn{8}{|l|}{ Loamy topsoil } \\
\hline \multirow{5}{*}{ Control } & 1,000 & $5.99(0.08)$ & $2.25(0.01)$ & 9.65 (1.07) & & & \\
\hline & 3,000 & $5.76(0.21)$ & $2.25(0.01)$ & $4.30(1.22)$ & & & \\
\hline & 8,000 & $5.99(0.10)$ & $2.17(0.01)$ & $1.56(0.36)$ & & & \\
\hline & 13,000 & $6.11(0.02)$ & $2.12(0.01)$ & $1.57(0.22)$ & & & \\
\hline & 18,000 & $6.11(0.07)$ & $2.19(0.02)$ & $0.63(0.11)$ & & & \\
\hline \multirow{5}{*}{ Fresh PyOM } & 1,000 & $6.75(0.06)$ & $2.36(0.03)$ & $21.14(1.96)$ & $0.106(0.002)$ & $596.4(46.6)$ & $2.9(0.1)$ \\
\hline & 3,000 & $6.26(0.12)$ & $2.16(0.01)$ & $2.50(0.16)$ & $0.070(0.009)$ & $45.3(4.2)$ & $1.9(0.2)$ \\
\hline & 8,000 & $6.21(0.08)$ & $2.15(0.01)$ & $1.21(0.14)$ & $0.031(0.001)$ & $9.8(1.2)$ & $0.8(0.1)$ \\
\hline & 13,000 & $6.06(0.12)$ & $2.19(0.01)$ & $2.01(0.61)$ & $0.008(0.005)$ & $6.1(4.4)$ & $0.2(0.1)$ \\
\hline & 18,000 & $6.21(0.07)$ & $2.19(0.01)$ & $1.25(0.25)$ & $N D$ & $N D$ & - \\
\hline \multirow{5}{*}{ Oxidized PyOM } & 1,000 & $6.45(0.09$ & $2.47(0.03)$ & $15.89(2.08)$ & $0.295(0.033)$ & $1,411.4(439.8)$ & $7.7(1.3)$ \\
\hline & 3,000 & $6.44(0.07)$ & $2.20(0.01)$ & $1.76(0.24)$ & $0.182(0.024)$ & $87.9(18.2)$ & $4.8(0.9)$ \\
\hline & 8,000 & $6.31(0.04)$ & $2.16(0.01)$ & $1.35(0.15)$ & $0.065(0.005)$ & $22.8(2.3)$ & $1.7(0.2)$ \\
\hline & 13,000 & $6.01(0.07)$ & $2.15(0.01)$ & $2.96(1.00)$ & $0.024(0.001)$ & $18.5(6.8)$ & $0.6(0.1)$ \\
\hline & 18,000 & $6.14(0.10)$ & $2.19(0.01)$ & $1.17(0.19)$ & $N D$ & $N D$ & - \\
\hline \multicolumn{8}{|l|}{ Loamy subsoil } \\
\hline \multirow{5}{*}{ Control } & 1,000 & $4.29(0.03)$ & $2.22(0.01)$ & $7.86(0.49)$ & & & \\
\hline & 3,000 & $4.35(0.04)$ & $2.23(0.01)$ & $1.22(0.16)$ & & & \\
\hline & 8,000 & $4.49(0.03)$ & $2.22(0.01)$ & $1.33(0.11)$ & & & \\
\hline & 13,000 & $4.64(0.07)$ & $2.23(0.01)$ & $0.74(0.07)$ & & & \\
\hline & 18,000 & $5.04(0.06)$ & $2.20(0.01)$ & $0.85(0.07)$ & & & \\
\hline \multirow{5}{*}{ Fresh PyOM } & 1,000 & $4.39(0.03)$ & $2.49(0.05)$ & $12.23(1.55)$ & $0.071(0.005)$ & $357.5(49.5)$ & $2.6(0.2)$ \\
\hline & 3,000 & $4.55(0.09)$ & $2.29(0.01)$ & $1.62(0.11)$ & $0.043(0.005)$ & $28.6(3.7)$ & $1.6(0.1)$ \\
\hline & 8,000 & $4.69(0.07)$ & $2.27(0.01)$ & $0.90(0.06)$ & $0.053(0.007)$ & $19.6(3.1)$ & $1.9(0.2)$ \\
\hline & 13,000 & $4.65(0.01)$ & $2.24(0.01)$ & $0.70(0.04)$ & $0.038(0.005)$ & $10.8(1.1)$ & $1.4(0.2)$ \\
\hline & 18,000 & $4.87(0.11)$ & $2.27(0.01)$ & $0.66(0.09)$ & $0.033(0.003)$ & $9.0(1.4)$ & $1.2(0.1)$ \\
\hline \multirow{5}{*}{ Oxidized PyOM } & 1,000 & $4.32(0.02)$ & $3.42(0.03)$ & $10.92(0.79)$ & $0.165(0.008)$ & $724.2(67.5)$ & $5.7(0.3)$ \\
\hline & 3,000 & $4.50(0.01)$ & $3.03(0.01)$ & $1.41(0.08)$ & $0.168(0.019)$ & $93.8(7.1)$ & $5.8(0.6)$ \\
\hline & 8,000 & $4.72(0.09)$ & $2.70(0.01)$ & $0.98(0.08)$ & $0.163(0.012)$ & $63.5(4.6)$ & $5.6(0.3)$ \\
\hline & 13,000 & $4.63(0.04)$ & $2.29(0.01)$ & $0.60(0.05)$ & $0.140(0.012)$ & $34.0(5.3)$ & $4.8(0.3)$ \\
\hline & 18,000 & $4.82(0.04)$ & $2.33(0.01)$ & $0.83(0.04)$ & $0.081(0.003)$ & $26.3(0.4)$ & $2.8(0.1)$ \\
\hline
\end{tabular}



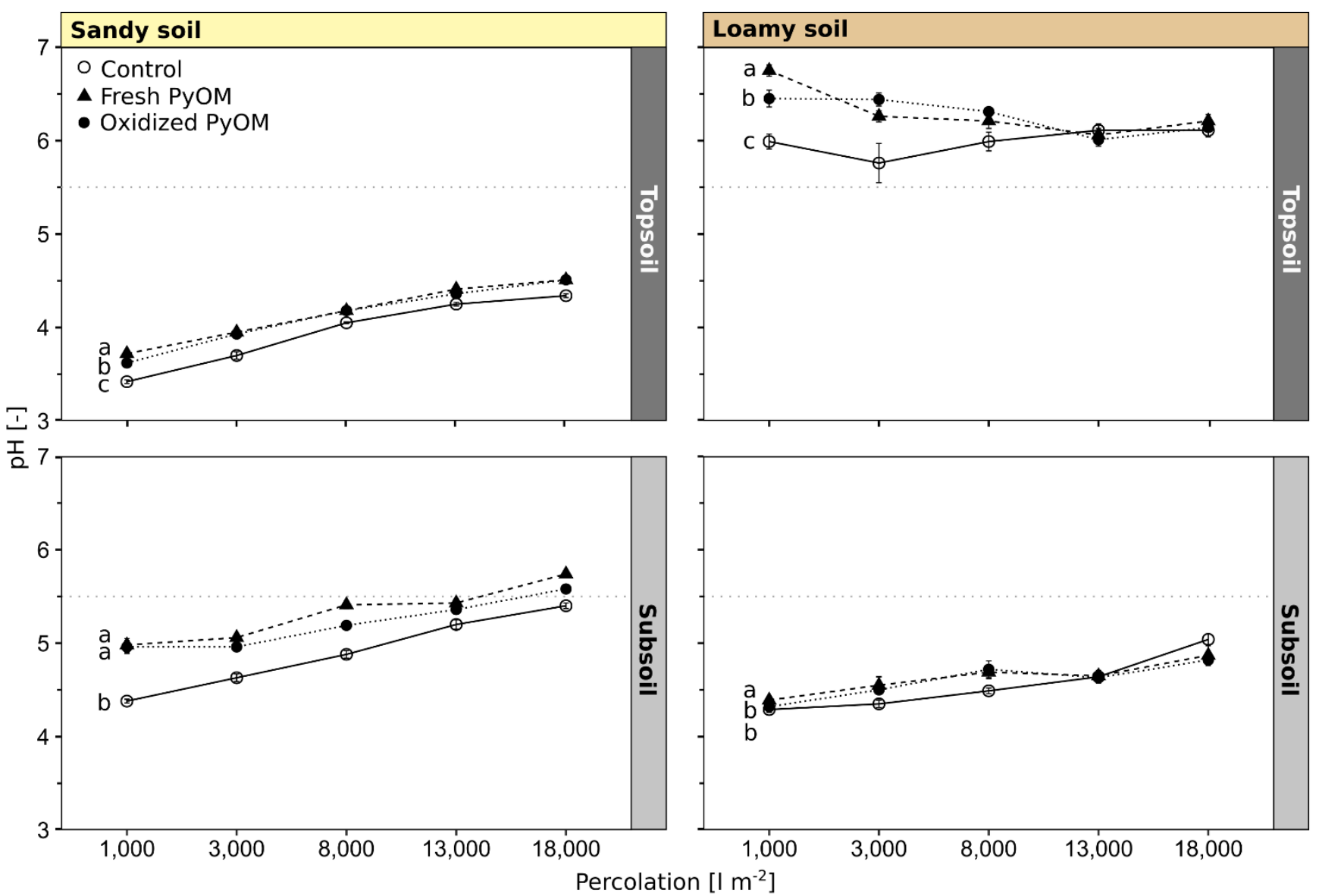

Figure S3: pH values of percolates from controls columns and columns with addition of fresh and oxidized PyOM percolated from topsoils and subsoils of the sandy and loamy soil $( \pm 1 \mathrm{SE})$. Significant differences in pH of the first flush $\left(1,0001 \mathrm{~m}^{-2}\right)$ are shown by lettering (p<0.05). The grey dotted line shows the background value $(\mathrm{pH}=5.5)$ of the $0.01 \mathrm{M}^{\mathrm{CaCl}} \mathrm{Cl}_{2}$ solution used for the percolation. 


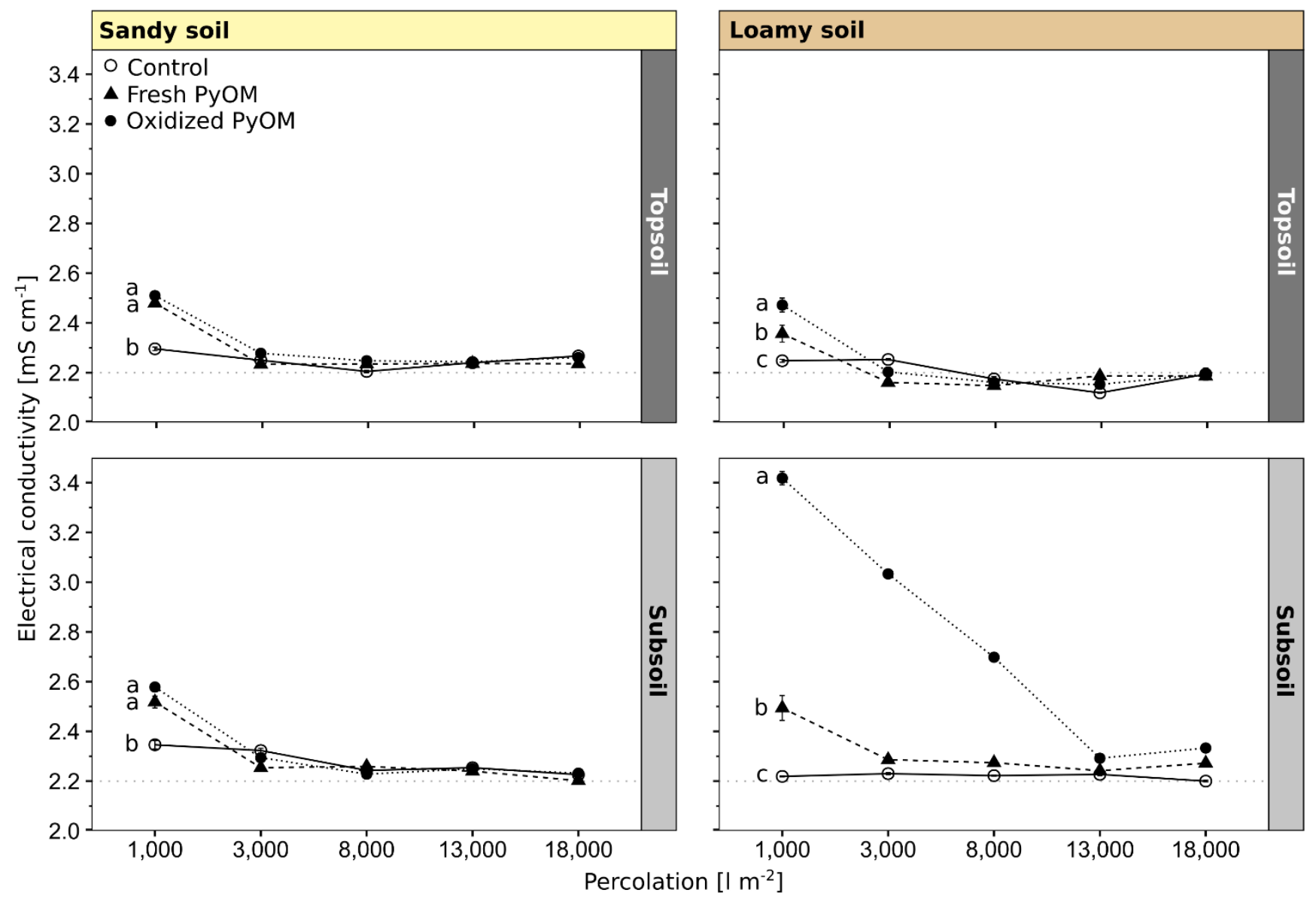

Figure S4: EC values of percolates from controls columns and columns with addition of fresh and oxidized PyOM percolated from topsoils and subsoils of the sandy and loamy soil $( \pm 1 \mathrm{SE})$. Significant differences in EC of the first flush $\left(1,0001 \mathrm{~m}^{-2}\right)$ are $\mathrm{shown}$ by lettering (p<0.05). The grey dotted line shows the background value $\left(\mathrm{EC}=2.2 \mathrm{mS} \mathrm{cm}^{-1}\right)$ of the $0.01 \mathrm{M}$ CaCl2 solution used for the percolation. 